\title{
RESEARCHES IN PRODUCING ORGANIC CERTIFIED SEEDS AND PLANTING MATERIAL
}

\author{
Anisoara Paun, Carmen Bracacescu, Dumitru Milea, George Bunduchi \\ National Institute of Research-Development for Machines and Installations Designed to \\ Agriculture and Food Industry, Romania \\ ani_paun@yahoo.com,carmenbraca@yahoo.com
}

\begin{abstract}
Obtaining satisfactory results in the production of seed and planting material, organic certified, depends to a great extent on the functional quality of the seed conditioning equipment and installations, but also on the quality of the staff involved in the production process. The paper presents the pilot installation for seed conditioning (which includes cleaning and sorting operations) and the experimental research in the establishment of its optimal operating parameters in order to reduce losses on processing chain links and increasing the production uses in the cereal and industrial plant seed precleaning process combined of two principles: counter flow aspiration of the product to be processed and separation on cylindrical sieves. The conclusion is that the sieve speed can be increased (also its productivity) without relative rest appearance by using certain special devices (interior cylindrical shields, inner inclined planes, interior cylindrical shields with brushes). Using this type of solutions, the speed can be increased 2-3 times compared to the speed of a simple cylindrical one thus also increasing its productivity.
\end{abstract}

Keywords: seeds, conditioning, cylindrical sieves.

\section{Introduction}

In ecological agriculture, the seed is used untreated, without herbicide, therefore a series of problems appear: cultures with lower purity because of the inefficient removal of foreign plants by weeding and hoeing and cultures with high incidence of specific pests.

These aspects require increasing the importance of seed pre-cleaning processes - removing from the product all foreign bodies or seeds by using a wide variety of pre-cleaning equipments.

Before receiving a specific destination (processing or planting), it is necessary that the harvested products undergo cleaning and sorting operations. Large farmers having cereal silos aim the elimination of impurities in the grain mass before storage, thus increasing the purity of the product, reducing transport and storage volume, maximum preservation of nutritional qualities for a long time and elimination of biological value characteristics impairments.

To reduce and even eliminate negative influences exerted by impurities in the grain mass it is necessary to perform precleaning using different machines or installations [1-6].

Keeping cereal seeds in optimal conditions in the storage systems requires the farmer to have included in the processing chain also high capacity machines for cleaning, sorting, storage, transport and equipment for drying cereal seeds $[7 ; 8]$

In order to improve the technology for cleaning and sorting cereal seeds at agricultural producers, to reduce losses on processing chain links (cleaning, sorting, storage, transport, processing itself, packaging, pre-commercial storage, distribution and marketing) and to ensure food safety, INMA Bucharest has developed a technology, Fig. 1, which led to the design and construction of a pilot installation for seed conditioning ICS, Fig. 2. It is composed of high capacity combined-type technical equipment (pre-cleaning module and cylindrical sieve). The project also aims to reach strategic objectives in the development of science, services, technologies and advanced applications in order to get in line with the priorities and objectives of national and European research and also to promote sustainable agriculture for improving the entire food chain ("from farm to fork").

\section{Materials and methods}

Seed conditioning installation ICS, Fig. 2, made up of the seed pre-cleaning module MPS-0 and cylindrical sieve SC-0 is designed to improve production technologies for different types of organic seed (cereal, legumes for grains, oilseeds, industrial plants and fodder, aromatic and medicinal plants, to solve practical problems concerning the production of organic seed for field cultures at farmers, in order to produce seed and planting material organic certified. 
Separation of impurities (raw foreign bodies and light bodies) of the seeds undergoing processing is performed using the pre-cleaning module MPS-0, Fig. 2, position 1, by means of two sieve drums and an intensive suction performed by a fan incorporated in the pre-cleaning module.

The cylindrical sieve SC-0, Fig. 2, position 2 is provided with four levelling blades, which register a continuous and regular movement of product mixing at the same time removing the vibrations.

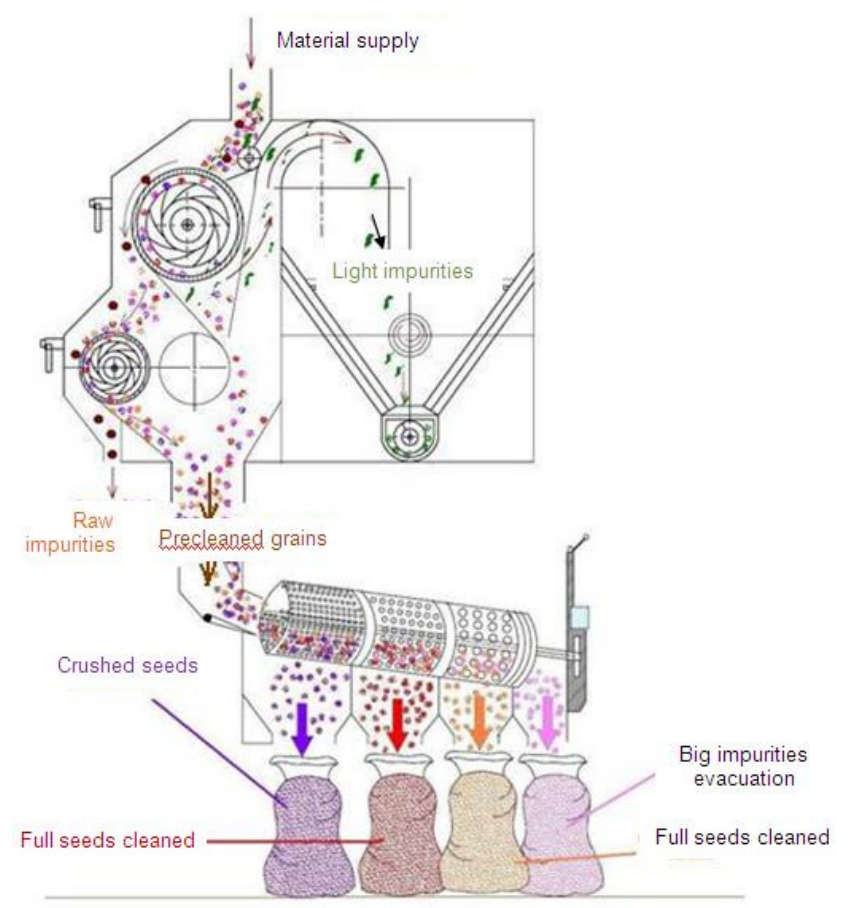

Fig. 1. Seed conditioning technology [9]

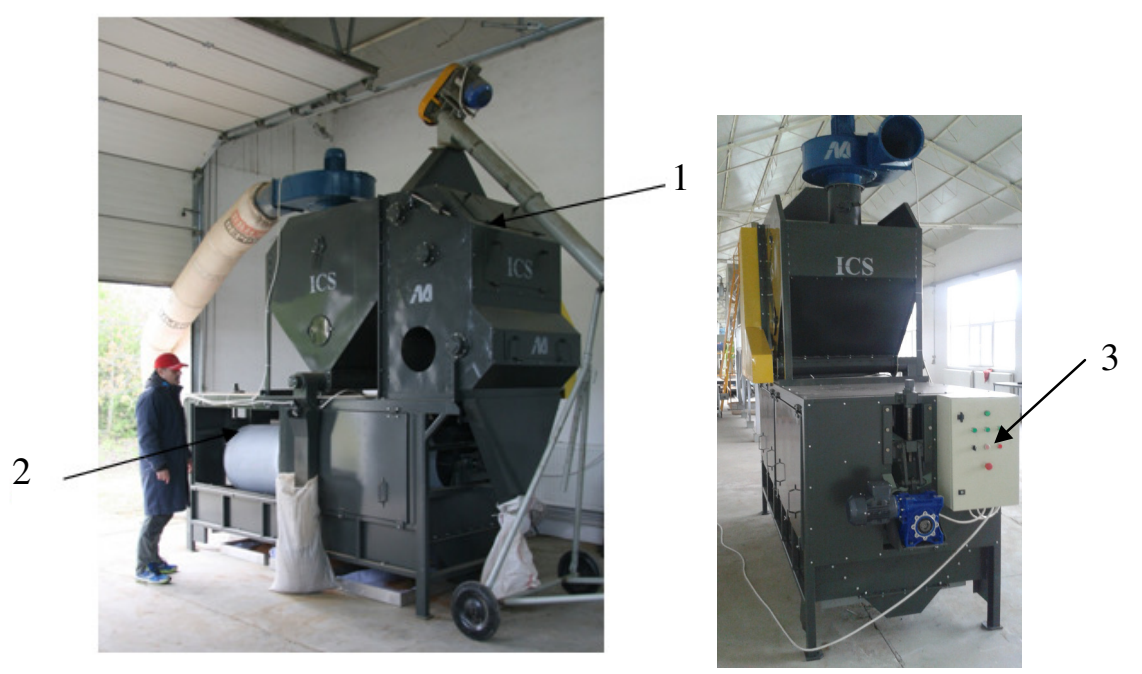

Fig. 2. Seed conditioning installation ICS: 1 - seed pre-cleaning module MPS-0; 2 - cylindrical sieve SC-0; 3 - drum tilting mechanism

The product, precleaned of raw foreign bodies and light impurities, reaches the cylindrical sieve where the separation in several fractions takes place. The sieve drum receives the rotation movement from a shaft gear motor. In the working process, the seeds with smaller size than the holes will go through them and those remaining as plus material will pass on the second sieve (if the cylindrical sieve is equipped with cylindrical sieves of different sizes, the smallest size being toward the supply). Some of them will pass through the holes of the second sieve (if they have the sorting size smaller than holes) and those that will remain as plus material will go to the third sieve. On this sieve the seeds having the sorting size smaller than the sieve holes will go through and the ones remaining as plus material will be evacuated through the discharge hopper. Due to the complete range of available 
selection sieves, the cylindrical sieve can be used with any type of seed. It should be noted that the selection of sieves that are installed on the sieve drum machine determines both the equipment capacity and the quality and quantity of the separated fractions. The equipment is provided with two air pressure and flow rate control systems to meet the suction conditions depending on the density of the primary product that is to be cleaned and on its purity. Sieve cleaning is made with a system of rollers. Supply flow control is achieved with the help of an automatic system, which commends opening and closing a shutter controlling seed mass penetration into the cylinder.

The main technical and functioning characteristics of the pilot installation ICS are:

Seed pre-cleaning module MPS-O:

- Productive capacity

For a standard product of type: $8 \mathrm{t} \cdot \mathrm{h}^{-1}$

- wheat, with hectolitre mass

$>75 \mathrm{~kg} \cdot \mathrm{hl}^{-1}$

- humidity

$6 . . .8 \%$

- cleaning degree

$40 . .50 \%$

Cylindrical sieve $S C-0$ :

- Selection capacity,

$3-4 t \cdot h^{-1}$

- Sieve rotation frequency,

$14 \mathrm{~min}^{-1}$

- Installed power

$0.75 \mathrm{~kW}$

The installation was carried out in the framework of an ongoing project, which foresees experiments throughout the year 2017. At this time, experiments were carried out only with the seed pre-cleaning module MPS under operating conditions, in order to determine the cereal seed precleaning degree, using as raw material wheat that was not pre-cleaned, purchased on the market. The experiments of the seed pre-cleaning module MPS were conducted according to accredited procedure [10] under operating conditions consisted in:

- Determinations of processed product and obtained products quality [7];

The samples were taken at the input and at the output of the pre-cleaning module and the following determinations were made:

- Product humidity;

- Physical purity, of which:

- Light foreign bodies (weeds, dust, husks, plant debris, peels+dry seeds being less than $1.5 \mathrm{~mm}$ thick) $\left(C_{\text {sui }}\right.$ and $\left.C_{\text {sue }}\right)$ :

- small foreign bodies ( $C_{s m i}$ and $C_{s m e}$ );

- large foreign bodies $\left(\mathrm{C}_{S M i}\right.$ and $\left.\mathrm{C}_{S M e}\right)$;

- ferromagnetic;

- hectolitre mass;

- percentage of good seeds in the product decanted by the cyclone.

- Determination of technological effect [7]

The technological effect of the seed pre-cleaning module MPS was analysed compared to the product standards and assessed on the basis of the results obtained in a single pass through the installation of the product to be processed. The following determinations were made:

- $E_{c s M}$ - large foreign bodies removed, \%;

- $E_{c s m}$ - small foreign bodies removed, \%;

- $E_{c s u}$ - light foreign bodies removed, \%;

- $C_{p s}$ - good seeds of the product to be processed lost in by-products, $\%$.

The calculation formulas for determining the technological effect of the pre-cleaning group used were [7]:

$$
\begin{aligned}
E_{c s M} & =\left[\left(C_{s M i}-C_{s M e}\right) / C_{s M i}\right] \times 100 \% \\
E_{c s m} & =\left[\left(C_{s m i}-C_{s m e}\right) / C_{s m i}\right] \times 100 \%
\end{aligned}
$$




$$
\begin{gathered}
E_{c s u}=\left[\left(C_{s u i}-C_{s u e}\right) / C_{s u i}\right] \times 100 \% \\
C_{p s}=\left(\Sigma m_{k} / M\right) \times 100 \%
\end{gathered}
$$

where $C_{S M i}-$ content of large foreign bodies at the module input, \%;

$C_{s M e}-$ content of large foreign bodies at the module output, \%;

$C_{s m i}$ - content of small foreign bodies at the module input, \%;

$C_{s m e}-$ content of small foreign bodies at the module output, \%;

$C_{\text {sui }}$ - content of light foreign bodies at the module input, \%;

$C_{\text {sue }}$ - content of light foreign bodies at the module output, \%;

$\Sigma m_{k}$ - sum of good seed masses, in the products collected at the module outputs, during the entire test and determined by laboratory analysis, based on the samples taken separately from each by-product, expressed as percentage reported to the total mass of the analysed sample;

$M$ - good seed mass at module input, determined by laboratory analysis based on the samples taken at the module input of product to be processed and expressed as percentage reported to the total mass of the samples.

\section{Results and discussion}

In the trials and measurements made in the experiments under operation conditions with the seed pre-cleaning module MPS the following data resulted presented in Tables 1, 2 and diagrams, Figure 3.

Table 1

\begin{tabular}{|c|c|c|c|c|c|}
\hline \multirow{2}{*}{$\begin{array}{l}\text { Crr. } \\
\text { No. }\end{array}$} & \multirow[b]{2}{*}{ Characteristic } & \multirow[b]{2}{*}{ UM } & \multirow[b]{2}{*}{ Test No. } & \multicolumn{2}{|c|}{ Value of parameter } \\
\hline & & & & $\begin{array}{c}\text { Input product in } \\
\text { equipment }\end{array}$ & $\begin{array}{c}\text { Exit product from } \\
\text { equipment }\end{array}$ \\
\hline 0 & 1 & 2 & 3 & 4 & 5 \\
\hline \multirow{4}{*}{1} & \multirow{4}{*}{ Physical purity } & \multirow{4}{*}{$\%$} & TI & 96.2 & 98.9 \\
\hline & & & TII & 94.8 & 99.1 \\
\hline & & & TIII & 95.1 & 98.8 \\
\hline & & & Mean & 95.37 & 98.93 \\
\hline \multirow{4}{*}{2} & \multirow{4}{*}{$\begin{array}{l}\text { Light foreign bodies } \\
\text { (thickness below } 1.5 \\
\mathrm{~mm}), C_{\text {sui }} \text { and } C_{\text {sue }}\end{array}$} & \multirow{4}{*}{$\%$} & $\mathrm{TI}$ & 0.72 & 0.19 \\
\hline & & & TII & 0.68 & 0.20 \\
\hline & & & TIII & 0.65 & 0.17 \\
\hline & & & Mean & 0.68 & 0.19 \\
\hline \multirow{4}{*}{3} & \multirow{4}{*}{$\begin{array}{l}\text { Large foreign bodies, } \\
C_{s M i} \text { and } C_{s M e}- \\
\text { collected at the exit } \\
\text { of the last drum }\end{array}$} & \multirow{4}{*}{$\%$} & TI & 1.7 & 0.2 \\
\hline & & & TII & 1.9 & 0.28 \\
\hline & & & TIII & 1.65 & 0.3 \\
\hline & & & Mean & 1.75 & 0.26 \\
\hline \multirow{4}{*}{4} & \multirow{4}{*}{ Chippings } & \multirow{4}{*}{$\%$} & TI & 1.5 & 0.2 \\
\hline & & & TII & 1.7 & 0.25 \\
\hline & & & TIII & 1.4 & 0.21 \\
\hline & & & Mean & 1.53 & 0.22 \\
\hline \multirow{4}{*}{5} & \multirow{4}{*}{ Humidity } & \multirow{4}{*}{$\%$} & TI & 7.6 & 7.4 \\
\hline & & & TII & 7.4 & 7.3 \\
\hline & & & TIII & 7.7 & 7.35 \\
\hline & & & Mean & 7.56 & 7.35 \\
\hline \multirow{4}{*}{6} & \multirow{4}{*}{$\begin{array}{l}\text { Small foreign bodies, } \\
C_{s m i} \text { and } C_{s m e}\end{array}$} & \multirow{4}{*}{$\%$} & TI & 0.22 & 0.04 \\
\hline & & & TII & 0.25 & 0.04 \\
\hline & & & TIII & 0.23 & 0.06 \\
\hline & & & Mean & 0.23 & 0.047 \\
\hline
\end{tabular}

Quality indices of the processed product 
Determining the losses coefficient of good seeds in byproducts

\begin{tabular}{|c|c|c|c|c|c|c|}
\hline \multirow{2}{*}{$\begin{array}{c}\text { Crr. } \\
\text { No. }\end{array}$} & Characteristic & \multirow{2}{*}{ UM } & \multicolumn{4}{|c|}{ Value of characteristic } \\
\cline { 4 - 7 } & & & T.I / 1 & T.II / & T.III / 3 & Mean \\
\hline 1 & Good seed mass at the entry into equipment, & $\%$ & 95.86 & 95.47 & 96.07 & 95.80 \\
\hline 2 & Good seed mass in light foreign bodies, $m_{1}$ & $\%$ & 0.08 & 0.05 & 0.09 & 0.07 \\
\hline 3 & Good seed mass at the exit of the cyclone, $m_{2}$ & $\%$ & 0.019 & 0.025 & 0.023 & 0.022 \\
\hline 4 & Sum of the masses of good seeds, $\Sigma m_{k}$ & $\%$ & 0.099 & 0.075 & 0.113 & 0.096 \\
\hline 5 & $\begin{array}{c}\text { Loss coefficient of good seeds in byproducts, } \\
C_{p s}\end{array}$ & $\%$ & 0.103 & 0.078 & 0.03 & 0.100 \\
\hline
\end{tabular}

Using relations 1,2, 3 and 4 and the data resulted in the experiments, the technological effect of the pre-cleaning group was determined:

$$
E_{c s M}=85.14 \% ; E_{c s m}=79.56 \% ; E_{c s u}=72.20 \% ; C_{p s}=0.1 \%
$$
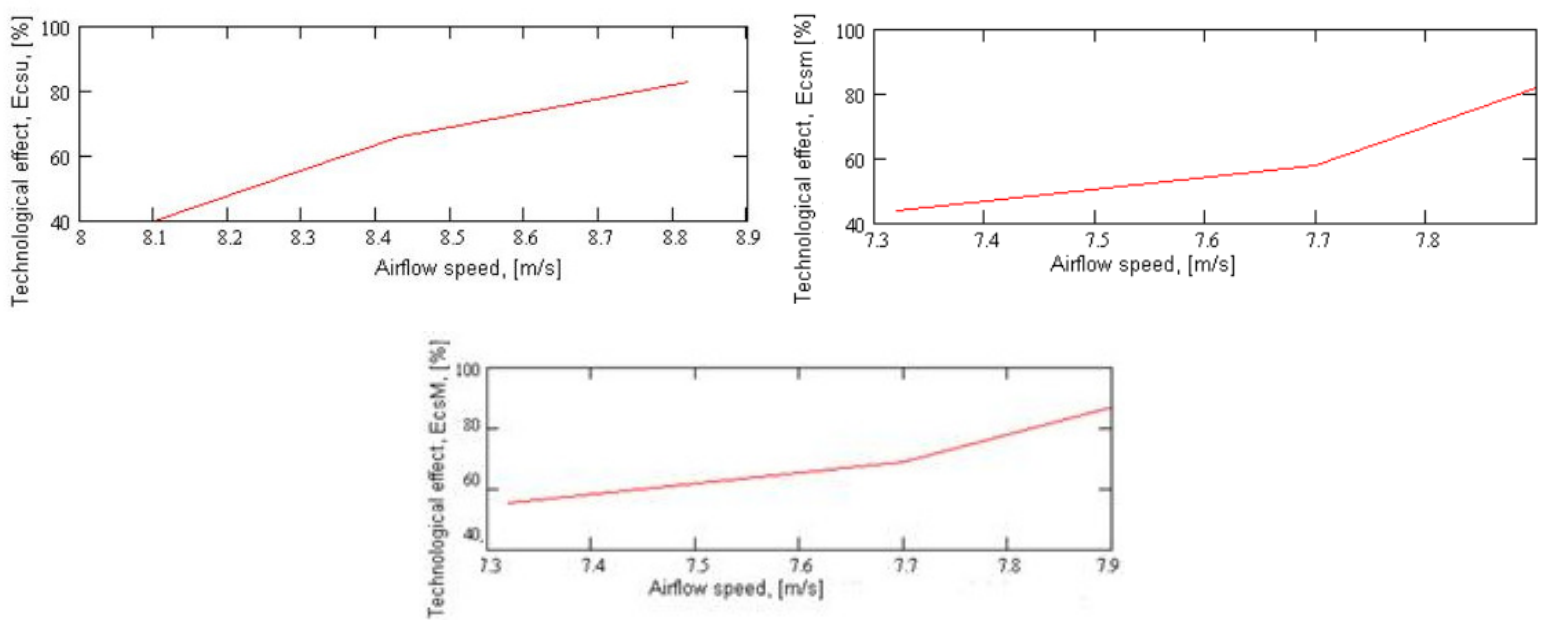

Fig. 3. Variation diagrams of $E_{c s u}, E_{c s m}, E_{c s M}$ according to the airflow speed

\section{Conclusions}

1. By increasing the air flow rate from up to $150 \mathrm{~m}^{3} \cdot \mathrm{min}^{-1}$ the technical separating installation performances are enhanced due to a better product layer structure achieved depending on the specific mass of particles;

2. The best technological effect resulted was of $85.14 \%$ for large foreign bodies eliminated;

3. The pre-cleaning module MPS-0 ensures an optimum drive mode of the seeds and impurities mass using two sieve drums, which can be replaced depending on the seeds undergoing precleaning.

\section{Acknowledgement}

This paper was financed with the support of ADER Project -ADER 1.2.2. Development of an integrated system for producing seed and planting material, organic certified, at field cultures: cereals, legumes for grains, oilseeds, industrial plants and fodder, aromatic and medicinal plants.

\section{References}

1. Brăcăcescu C., Găgeanu I., Popescu S., Kemal C.S. Researches concerning impurities separation process from mass of cereal seeds using vibrating sieves in air flow currents, in Proceedings of 5th International Scientific Conference "Engineering for rural development 2016" Jelgava, Letonia , ISSN1691-5976, pp.364-370. 
2. Căsăndroiu T. Primary processing and agricultural products storing, lithographed course, PolitehnicaUniversity of Bucharest, 1993, $176 \mathrm{p}$.

3. Ciobanu V.G., Vişan A.L., Păun A, Nedelcu A. Comparative study regarding seed sorting equipment and the importance of implementing smart systems within the working process, JoKULL Journal 2015, Vol.64, No.9, ISSN 0449-0576, pp. 91-100.

4. Găgeanu P. Study on corn seeds sorting, Doctoral Thesis, UPB-ISB, 2001, 185 p.

5. Yan J., Liu C., Zhao L. Dynamic characteristics of vibrating screen with determinate structure and statistically indeterminate structure, Applied Mechanics and Materials 34-35, 2010, pp.1850-1854

6. Târcolea C., Căsăndroiu T., Voicu Gh. Stochastic models for simulating seed separation process on sieves, in Proceedings of the 36th International Symposium "Actual Tasks on Agricultural Engineeering", 2008, Croatia, pp.293-306.

7. Păun A., Pirnă I., Găgeanu P., Vlăduţ V. Increasing the added value of processed products in the milling industry by implementing a combined calibrator in wheat preparation technological scheme, INMATEH - Agricultural Engineering, vol. 36, no. 1/2012, Bucharest - Romania, , e: ISSN: 2068 - 2239; p: ISSN: 2068-4215, pp. 63-68.

8. Voicu Gh., Orăşanu N. Considerations regarding the study on particle motion on the sieve plan with circular motion, Works of the second International Conference "Research People and Actual Tasks on Multidisciplinary Sciences", volume 2, Lozenec, Bulgaria, June 10-12, 2009.

9. Păun A., Ionita Gh., Milea D., Ganea -Christu I. - Patent application - Seed conditioning technology, no. A/00887/2016;

10. Testing Department - General testing procedure PGI-01.20 - Pre-cleaning and cleaning installations for cereals and technical plants, INMA Bucharest, 2012. 